\title{
Messengers and Messages for Tweets That Used \#thinspo and \#fitspo Hashtags in 2016
}

\author{
Jenine K. Harris, $\mathrm{PhD}^{1}$; Alexis Duncan, $\mathrm{PhD}^{1,2}$; Vera Men, $\mathrm{BS}^{1}$; Nora Shevick, $\mathrm{BS}^{3}$; \\ Melissa J. Krauss, $\mathrm{MPH}^{2}$; Patricia A. Cavazos-Rehg, $\mathrm{PhD}^{2}$
}

Accessible Version: www.cdc.gov/pcd/issues/2018/17_0309.htm

Suggested citation for this article: Harris JK, Duncan A, Men V, Shevick N, Krauss MJ, Cavazos-Rehg PA. Messengers and Messages for Tweets That Used \#thinspo and \#fitspo Hashtags in 2016. Prev Chronic Dis 2018;15:170309. DOI: https://doi.org/ $10.5888 /$ pcd15.170309.

\section{PEER REVIEWED}

\section{Abstract}

\section{Introduction}

Twitter is widely used by young adults and is popular for seeking and sharing health information. The hashtags \#thinspo and \#fitspo provide a way to identify tweets designed to inspire thinness (thinspiration, thinspo) or fitness (fitspiration, fitspo). However, despite having different purposes, both terms may be associated with content that promotes eating disorders. We sought to 1) examine and compare the characteristics of senders and the content of tweets using these hashtags and 2) identify characteristics associated with engagement with a \#thinspo or \#fitspo tweet.

\section{Methods}

In May 2016 we collected 1,035 tweets with \#thinspo and \#fitspo hashtags by using a constructed week sampling procedure. Using consensus coding, pairs of raters assessed each tweet's topic and associated images and videos. We used descriptive statistics to examine topics and user characteristics and inferential models to determine topics and characteristics associated with retweets, likes, and replies to tweets.

\section{Results}

Of the 1,035 tweets, $696(67.2 \%)$ were relevant to body image, fitness, food, dieting, or eating disorders. Fitspo tweets came from organizations or businesses, were promotional, and focused on nutrition and exercise, whereas \#thinspo tweets came from individuals, focused on thinness and disordered eating behaviors, and con- tained images of extremely thin women. Rates of retweeting and liking were significantly higher for \#thinspo than for \#fitspo.

\section{Conclusion}

Characteristics of messages and messengers differed between \#thinspo and \#fitspo tweets; \#thinspo tweets were used for messages about disordered eating. Public health professionals should consider using the \#thinspo hashtag to reach the \#thinspo group.

\section{Introduction}

Disordered eating behaviors are common, particularly among adolescent and young adult women, with rates in these groups as high as $16 \%$ for binge eating, $20 \%$ for purging compensatory behaviors (vomiting, laxatives, diuretics, diet pills), and $61 \%$ for nonpurging compensatory behaviors (fasting, skipping meals, restricting) $(1,2)$. These behaviors are associated with leading causes of morbidity and mortality, including depression, alcohol misuse, and obesity $(1,3)$. The age of onset for eating disorders is typically the late teens or early twenties (4).

As of $2015,89 \%$ of US teenagers aged 13 to 17 and $86 \%$ of US adults aged 18 to 29 used social media; $33 \%$ of 13 - to 17 -year-olds and $36 \%$ of online 18 - to 29 -year-olds used Twitter $(5,6)$. The percentages of teenaged and young adult Twitter users who are males and nonwhite are higher than percentages of these groups in the general population, and young adults who use Twitter are more likely to be college graduates than young adults in the general population $(6,7)$. Twitter is an application for "microblogging," or sending and receiving brief (140 characters) messages or "tweets." Twitter accounts can be followed by other Twitter users, allowing individuals or organizations to receive and share or "retweet" messages to followers. Twitter users can also "like" a tweet by clicking on a heart, enter a reply that will show beneath the original tweet if clicked on, or mention another Twitter user by including @ username in a tweet; the tweet with the mention will then appear in the mentioned account. Hashtags are used on Twitter for the formation of groups interested in particular topics or events (8) and make it easier to find tweets and like or share them (9). 
Facebook is more widely used than Twitter, and older adults and parents are less likely to monitor the use of Twitter by adolescents and young adults than they are to monitor use of Facebook $(10,11)$, affording young users greater anonymity. This anonymity may increase sharing of information on Twitter on such health issues as disordered eating behaviors or struggles with body image (12). Evidence of changes in health behaviors resulting from exposure to and use of social media is mixed $(13,14)$; however, approximately $80 \%$ of US adult internet users have searched online for health information $(15,16)$ and $70 \%$ report being influenced by what they found (17).

The quality of health information on social media varies widely; one study found that about half of "healthy living" blogs contained negative and stigmatizing messages about food or weight (18). Because of high rates of social media use among adolescents and young adults, concern is increasing about the promotion of disordered eating behaviors among online communities $(19,20)$. Viewing images of extremely thin people may alter an individual's views on healthy body image and weight and contribute to normalization of disordered eating behaviors (21). Social media users with disordered eating behaviors who feel isolated may be especially vulnerable to these messages as they seek a sense of belonging (22).

Thinspiration and thinspo are terms used on social media to identify messages as inspiration for weight loss $(23,24)$. Likewise, fitspiration and fitspo are used when posting messages to inspire people to exercise and eat healthy food (24-26). Although intended to focus on healthy behavior, fitspo has been described as similar to thinspo in promoting unhealthy behaviors $(24,25)$. To better understand social media users who send messages and engage with \#thinspo and \#fitspo, we 1) examined and compared senders and content of tweets using the \#thinspo and \#fitspo hashtags, and 2) identified characteristics associated with engagement with \#thinspo and \#fitspo tweets.

\section{Methods}

In May 2016 we used a constructed week sampling procedure $(27,28)$ to collect tweets that included \#thinspo or \#fitspo. The constructed week includes one Monday, one Tuesday, and so forth during a specified time to account for variation in volume and topics on different days and during different times of a month. We initially collected data on 2 constructed weeks (14 days) of tweets $(\mathrm{n}=28,941)$ for an average of 2,067 \#thinspo or \#fitspo tweets daily. From the 2 weeks we selected a single week and took a random sample of 100 \#thinspo and 50 \#fitspo tweets per day for coding. We used \#thinspo and \#fitspo instead of \#thinspiration and \#fitspiration after an informal review of hashtags found the short- er versions were used more often. We sampled fewer tweets from \#fitspo after we found \#thinspo tweets were more commonly sent. For each tweet, we collected data on the number of followers of the sender, the number of users followed by the sender, and the number of tweets the sender had ever sent. To determine engagement with the \#thinspo and \#fitspo tweets, approximately one year after the tweets in our data were sent, we collected the number of retweets, likes, and replies to each tweet.

Initial data were collected by using NodeXL (Social Media Research Foundation) (29), a plug-in for Excel (Microsoft Corp) that accesses Twitter and downloads tweets based on key words, hashtags, Twitter user names, and other information. To collect the number of retweets, likes, and replies, we opened each tweet manually and recorded the information. The sampling process resulted in 700 tweets collected with the \#thinspo hashtag and 354 tweets collected with the \#fitspo hashtag. A small number $(n=18)$ of duplicate tweets with both hashtags and one tweet with neither hashtag were removed. The final sample size for coding was 1,035 unique tweets: 692 \#thinspo tweets and 343 \#fitspo tweets from 562 unique Twitter user accounts.

\section{Coding tweets}

The codebook was developed by using an iterative process. We started the codebook by including codes consistent with related studies examining thinspiration or fitspiration social media content (24-26). The initial codebook included items related to the focus of the tweet text and the focus of images, videos, and websites linked from the tweet. We also included codes for whether the tweet appeared promotional (ie, promoting a product, organization, or business), whether it was in English, whether the text of the tweet was only hashtags, and whether the Twitter account the tweet came from appeared to belong to a person or an organization or business. After 3 authors (A.D., N.S., V.M.) coded a sample of 50 tweets, we met to discuss modifications to the codebook. The modified codebook was then used by the 3 authors for 2 rounds of reliability coding on approximately $5 \%$ of the data; after each round, the authors held a group discussion to clarify codes with low reliability. The Fleiss $\kappa$ for the final round of reliability coding was almost perfect (0.81-1.0) for 17 of the 43 codes, fair to substantial agreement $(0.41-0.80)$ for 11 codes, and below 0.41 for 15 codes. Because we did not reach at least moderate agreement for all codes, for the final coding we used consensus coding, whereby 2 coders classified each tweet and met to discuss and arrive at consensus. Each of 3 coders was assigned approximately two-thirds of the tweets; each tweet was coded by 2 coders.

After reliability testing and discussion, the following 10 final text topic codes were included: eating less, eating healthy, exercise, gaining strength, losing weight, binging, purging (vomiting or us-

\footnotetext{
The opinions expressed by authors contributing to this journal do not necessarily reflect the opinions of the U.S. Department of Health and Human Services, the Public Health Service, the Centers for Disease Control and Prevention, or the authors' affiliated institutions.
} 
ing laxatives or diuretics), wanting a body type or body part characteristic (eg, thigh gap), mentions of a type of eating disorder, and mentions of a medication. Each tweet could be coded for more than one topic. Image and video codes were as follows: images or video focuses on food, body or parts of a body, or something else; number of images or videos; number of images or videos that show a male, a female, or "can't tell [sex of person shown]"; number of images or videos that show a person who is extremely skinny or skeletal, muscular, or neither; number of images or videos that show butt, thighs-legs, arms, neck-shoulders, torso (shoulders to hips), waist-stomach-hips, or full body; number of images that show healthy food like fruits, vegetables, grilled meat, or unhealthy food like candy, chips, fried food, or a combination of healthy and unhealthy food. Web URLs in tweets were clicked and the main topic of the web page was coded as dieting, healthy eating, physical activity, medication-vitamins-supplements, something else. Mentions were coded by searching each tweet for the@symbol and coding tweets with@ as including a mention.

\section{Data analysis}

The 562 unique Twitter users in the 1,035 tweet data set included 403 users (71.7\%) who contributed one tweet and 159 users who contributed 2 to 23 tweets. Because of multiple tweets from 159 Twitter users, the data failed the assumption of independent observations, so we used descriptive statistics to examine topics and characteristics for all tweets and to compare \#thinspo and \#fitspo tweets.

Few tweets included an image in any one category (eg, torso, arms), resulting in medians for the number of images per category being 0 or near 0 . We recoded the number of images variables to binary variables for whether or not each tweet included each image category and reported frequencies and percentages for tweets containing each type of image.

To determine which topics and characteristics were associated with retweets, likes, and replies, we examined the distribution of these variables and selected an appropriate statistical model. All 3 variables failed a Shapiro-Wilks test of normality. Just 94 tweets had any replies, and the distributions of the retweet and likes variables were extremely right skewed with variances far above their means. We recoded the replies variable as a binary variable: no replies and one or more replies.

Because past research demonstrated that the characteristics of Twitter users influence retweeting, liking, and replying to tweets (9), we used 2-level models with tweet characteristics at level 1 and Twitter user characteristics at level 2. We used 2-level negative binomial regression to predict retweeting and likes and 2-level logistic regression to predict replies. Models included Twitter user-level and tweet-level characteristics associated with retweeting, liking, and replying to tweets (ie, engaging with tweets) in prior studies, including number of tweets and followers (9) and the type of Twitter user (30). Although hashtag and URL inclusion in a tweet influences engagement (9), all tweets included at least one hashtag because of the method of data collection, and none of the \#thinspo tweets included URLs, so we did not include hashtag or URL variables in the models. There is no evidence we are aware of that including an image or video in a tweet increases engagement, so we did not include this variable in the models. There is mixed evidence on whether including a mention decreases engagement (31) or has no influence (9) on engagement, so we included mentions in the model. Finally, we added a predictor representing whether the tweet included \#thinspo or \#fitspo. We used Akaike information criterion (AIC) to compare model fit and interpreted better-fitting models (ie, models with lower AIC). Clean data and $\mathrm{R}$ commands for analyses are available at https:/github.com/ coding2share/Research-project-code/tree/master/thinspo-fitspo. Alpha was set at .05. The study was approved by the institutional review board at Washington University in St. Louis (IRB 201611145).

\section{Results}

Of the 1,035 tweets, 696 were relevant to body image, fitness, food, dieting, or eating disorders; 15 tweets were not relevant and 324 were inaccessible $(n=315)$ or had content that was unclear ( $n$ =9). Most relevant tweets were in English (Table 1). Thinspo tweets had higher medians than \#fitspo tweets for numbers of retweets and likes. Only $94(13.5 \%)$ tweets received replies: $17.9 \%$ of \#thinspo tweets and $5.0 \%$ of \#fitspo tweets. Of 458 \#thinspo tweets, 141 (30.8\%) were hashtags only, while 21 of 238 (8.8\%) \#fitspo tweets were hashtags only. Most (98.1\%) hashtagsonly tweets included images or videos.

More than half of \#fitspo tweets were promotional and/or included a link to a website, while just one \#thinspo tweet was promotional, and none included a link to a website. A small number $(\mathrm{n}=32)$ of \#fitspo tweets focused on food images, while no \#thinspo tweets focused on food images. Most (95.0\%) \#thinspo tweets included images or videos showing a body or body part; only $47.9 \%$ of \#fitspo tweets did so. About one-third (33.8\%) of \#thinspo tweets included one or more images or videos focused on the waist-stomach-hips, while only 3.4\% of \#fitspo tweet images/ videos did so. Most (75.5\%) \#thinspo tweets included an image showing an extremely skinny or skeletal person; only $3.4 \%$ of \#fitspo tweets included such an image. In addition, $18.9 \%$ of \#fitspo tweets included images/videos of a muscular person, while muscular people were featured in $0.7 \%$ of \#thinspo tweets. Of the 45 \#fitspo tweets with muscular images, 26 were of females only, 13

The opinions expressed by authors contributing to this journal do not necessarily reflect the opinions of the U.S. Department of Health and Human Services, the Public Health Service, the Centers for Disease Control and Prevention, or the authors' affiliated institutions. 
images were of males only (out of the 15 \#fitspo tweets with males-only images), 5 were of both males and females, and one image was of a body that was not clearly male or female. Nearly all (95\%) \#thinspo tweet images were of females (Table 1).

The percentages of tweets on each topic differed for \#thinspo and \#fitspo (Figure). Compared to \#thinspo tweets, a higher percentage of \#fitspo tweets contained the topics of exercise, strength, eating less, eating healthy, and medication. \#Thinspo tweets had higher percentages than \#fitspo tweets of the topics of losing weight, wanting a certain body type or body part characteristic, eating disorders, binging, and purging.

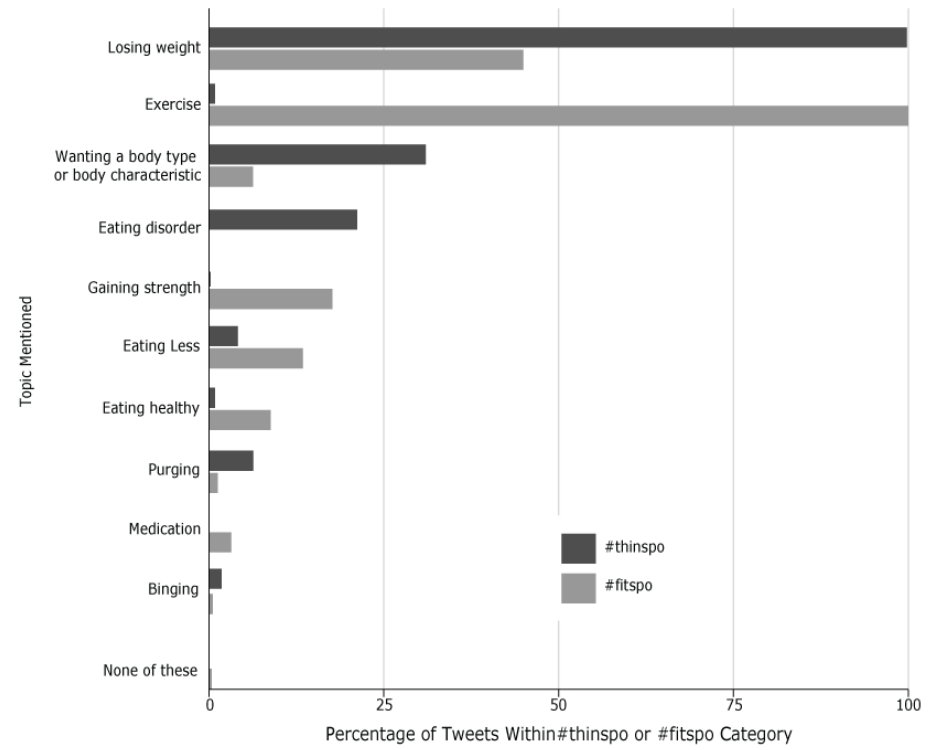

Figure. Percentage of topics mentioned in a sample of \#thinspo or \#fitspo tweets, May 2016. Percentages add to more than 100 because each tweet could be coded for multiple topics.

The \#fitspo tweeters had nearly twice the number of followers that \#thinspo tweeters had (Table 2). Likewise, \#fitspo tweeters tweeted more overall than did \#thinspo tweeters, while \#thinspo tweeters favorited more tweets (Table 2). Some of these differences may be explained by the higher percentage of \#fitspo tweeters $(54.4 \%)$ than \#thinspo tweeters $(0.3 \%)$ who appeared to represent organizations or businesses; a greater percentage of \#thinspo tweeters (99.7\%) than \#fitspo tweeters (35.0\%) appeared to be individuals.

The retweet model with the \#thinspo-\#fitspo variable (AIC = $3,694.6$ ) was a better fit than the model without ( $\mathrm{AIC}=3,813.6$ ). In the better-fitting model, retweets were positively and significantly associated with tweeting \#thinspo compared with \#fitspo
(Table 3). The Twitter user appearing to be an individual representing personal views was also positively and significantly associated with retweets, while the number of tweets from the account was negatively and significantly associated with retweets. The number of followers and the "unable to tell" user type were not associated with retweets.

The likes model with the \#thinspo-\#fitspo variable was a better fit (AIC $=4,055.2)$ than the model without the \#thinspo-\#fitspo variable $(\mathrm{AIC}=4,125.8)$. Consistent with the retweet model, the "unable to tell" user type and the number of followers were not significantly associated with likes; however, using the \#thinspo hashtag and appearing to be an individual representing personal views were both positively and significantly associated with the number of likes (Table 3). The number of tweets sent by the Twitter user was negatively and significantly associated with likes. Finally, the model without the \#thinspo-\#fitspo variable was a slightly better fit $(\mathrm{AIC}=359.6)$ than the same model with the \#thinspo-\#fitspo variable $(\mathrm{AIC}=361.5)$ at explaining replies. However, none of the predictors were significant in either model (Table 3 ).

\section{Discussion}

We examined 1,035 tweets that used the \#thinspo and \#fitspo hashtags from a constructed week in May 2016. Although a recent study found similarities in content on thinspiration and fitspiration websites (24), we found differences in messages, messengers, and engagement between tweets containing \#thinspo and tweets containing \#fitspo.

The topics of \#thinspo tweets were different from the topics of \#fitspo tweets: \#fitspo tweets were more likely to include the topics of exercise, strength, healthy eating, eating less, and medication and \#thinspo tweets were more likely to include the topics of binging, purging, losing weight, eating disorders, and wanting a certain body type or body part characteristic.

In addition to having different messages, \#thinspo and \#fitspo tweets had differences in messengers. Consistent with the promotional content, \#fitspo tweets were more likely than \#thinspo tweets to be sent by organizations or businesses or people representing them. With only one exception, \#thinspo tweets were sent by accounts that appeared to belong to an individual representing personal views. Also consistent with \#fitspo tweeters being organizations or businesses, those who sent \#fitspo tweets had more followers and sent more tweets than those who sent \#thinspo tweets.

We also found differences in engagement: \#thinspo tweets had significantly more retweets and likes than \#fitspo tweets, which is contrary to expectations and prior research (9), given the larger number of followers and tweets sent by \#fitspo tweeters. Even

\footnotetext{
The opinions expressed by authors contributing to this journal do not necessarily reflect the opinions of the U.S. Department of Health and Human Services, the Public Health Service, the Centers for Disease Control and Prevention, or the authors' affiliated institutions.
} 
after the \#thinspo-\#fitspo variable was added to the model, the number of followers of a Twitter user was negatively associated with likes, suggesting that people using these hashtags are liking tweets from individuals rather than from organizations or businesses. Taken together with the characteristics of the messenger, it appears that Twitter users using the \#thinspo hashtag may be a community of individuals coalescing around the thin body image while \#fitspo tweeters are businesses promoting or selling fitnessrelated products.

Although the link between social media consumption and health behavior is not yet clear, engaging with social media content through retweeting or liking a tweet may have some influence on behavior $(13,14)$. The \#thinspo tweets were positively and significantly associated with retweets and likes, and the content focused on unhealthy behaviors and images. Meanwhile, \#thinspo tweeters mostly represented personal views. These characteristics suggest an engaged community sharing information that normalizes disordered eating behaviors (21) and increases body dissatisfaction and disordered eating behaviors (23). Although eating disorder-focused advocacy organizations, research centers, and treatment facilities are active on Twitter, they do not often use hashtags in their messages. Our findings suggest that these organizations may reach more people in need of intervention for disordered eating behaviors if they use the \#thinspo hashtag. Organizations may also wish to consider a strategy in which employees tweet from separate accounts to take advantage of higher levels of engagement with individual accounts compared with organizational accounts.

Our study has several limitations. First, our data collection strategy included \#thinspo and \#fitspo, which may have excluded tweets with the longer \#thinspiration and \#fitspiration hashtags. In addition, although the constructed week strategy of collecting tweets increased the likelihood that our data were representative, the selected tweets may not be representative of the population of \#thinspo and \#fitspo tweets. Despite these limitations, this study contributes to our understanding of how \#thinspo and \#fitspo are used on social media by identifying differences in the messengers, messages, and engagement that could aid public health practitioners and researchers in identifying and contacting people at risk for disordered eating behavior. Future studies might examine whether changes in knowledge, attitudes, or behaviors would result from sending replies or direct messages about healthy eating or positive body image to Twitter users who tweet the \#thinspo hashtag.

\section{Acknowledgments}

This study was funded by the National Institutes of Health (R01 DA039456), the National Institute of Mental Health (R21 MH112331), and a Robert Wood Johnson Foundation award for promoting open science. The authors have no conflicts of interest.

\section{Author Information}

Corresponding Author: Jenine K. Harris, PhD, Brown School, Washington University in St. Louis, One Brookings Dr, Campus Box 1196, St. Louis, MO 63130. Telephone: 314-935-3522. Email: harrisj@wustl.edu.

Author Affiliations: ${ }^{1}$ Brown School, Washington University in St. Louis, St. Louis, Missouri. ${ }^{2}$ Department of Psychiatry, School of Medicine, Washington University in St. Louis, St. Louis, Missouri. ${ }^{3}$ Washington University in St. Louis, St. Louis, Missouri.

\section{References}

1. Solmi M, Veronese N, Favaro A, Santonastaso P, Manzato E, Sergi $\mathrm{G}$, et al. Inflammatory cytokines and anorexia nervosa: a meta-analysis of cross-sectional and longitudinal studies. Psychoneuroendocrinology 2015;51:237-52.

2. Neumark-Sztainer D, Wall M, Larson NI, Eisenberg ME, Loth $\mathrm{K}$. Dieting and disordered eating behaviors from adolescence to young adulthood: findings from a 10-year longitudinal study. J Am Diet Assoc 2011;111(7):1004-11.

3. Field AE, Sonneville KR, Micali N, Crosby RD, Swanson SA, Laird NM, et al. Prospective association of common eating disorders and adverse outcomes. Pediatrics 2012; 130(2):e289-95.

4. Hilbert A, Pike KM, Goldschmidt AB, Wilfley DE, Fairburn CG, Dohm FA, et al. Risk factors across the eating disorders. Psychiatry Res 2014;220(1-2):500-6.

5. Perrin A, Duggan M, Greenwood S. Social media update 2016. http://www.pewinternet.org/2016/11/11/social-media-update2016. [Archived by WebCite at http://www.webcitation.org/ 6r4JgyNV8.] Accessed January 11, 2017.

6. Lenhart A. Internet \& Technology: Teens, social media \& technology overview 2015. Mobile access shifts social media use and other online activities. Washington (DC): Pew Research Center; April 9, 2015. http://www.pewinternet.org/ 2015/04/09/mobile-access-shifts-social-media-use-and-otheronline-activities/. Accessed December 4, 2017.

\footnotetext{
The opinions expressed by authors contributing to this journal do not necessarily reflect the opinions of the U.S. Department of Health and Human Services, the Public Health Service, the Centers for Disease Control and Prevention, or the authors' affiliated institutions.
} 
7. American FactFinder. https://factfinder.census.gov/faces/nav/ jsf/pages/index.xhtml. Accessed August 23, 2017.

8. Bruns A, Burgess JE. The use of Twitter hashtags in the formation of ad hoc publics. Proceedings of the 6th European Consortium for Political Research (ECPR) General Conference 2011; 2011, Aug 25-27; Reykjavik, Iceland. [Archived by WebCite at http://www.webcitation.org/6QNK93vep]. Accessed January 11, 2017.

9. Suh B, Hong L, Pirolli P, Chi EH. Want to be retweeted? Large scale analytics on factors impacting retweet in twitter network. Institute of Electrical and Electronics Engineers Second International Conference on Social Computing (SocialCom) Kirkkonummi, Finland, Aug 20-22, 2010:177-184.

10. De Cristofaro E, Soriente C, Tsudik G, Williams A. Hummingbird: privacy at the time of Twitter. Institute of Electrical and Electronics Engineers; 2012:285-299. https:// eprint.iacr.org/2011/640.pdf.[Archived by WebCite at http:// www.webcitation.org/6r4Ku4Aku.] Accessed January 11, 2017.

11. Bennett S. Twitter USA: 48.2 million users now, reaching 20\% of population by 2018. AdWeek. 2014. http:// www.adweek.com/digital/twitter-usa-users/. [Archived by WebCite at http://www.webcitation.org/6r4G2E3zM.] Accessed June 8, 2017.

12. 12. National Association of Anorexia Nervosa and Associated Disorders. Eating disorders statistics. http://www.anad.org/getinformation/about-eating-disorders/eating-disorders-statistics/. [Archived by WebCite at http://www.webcitation.org/ 6r4G5Onr0.] Accessed March 30, 2016.

13. Chou WY, Prestin A, Lyons C, Wen KY. Web 2.0 for health promotion: reviewing the current evidence. Am J Public Health 2013;103(1):e9-18.

14. Maher CA, Lewis LK, Ferrar K, Marshall S, De Bourdeaudhuij I, Vandelanotte $\mathrm{C}$. Are health behavior change interventions that use online social networks effective? A systematic review. J Med Internet Res 2014;16(2):e40.

15. Moorhead SA, Hazlett DE, Harrison L, Carroll JK, Irwin A, Hoving C. A new dimension of health care: systematic review of the uses, benefits, and limitations of social media for health communication. J Med Internet Res 2013;15(4):e85.

16. Fox S. The social life of health information. http:// www.pewinternet.org/Reports/2011/Social-Life-of-HealthInfo.aspx. [Archived by WebCite at http:// www.webcitation.org/6r4GE7o86.] Accessed July 10, 2016.
17. Fox S, Rainie L. The online health care revolution: how the web helps Americans take better care of themselves. Washington (DC): Pew Internet and American Life Project; 2000.

18. Boepple L, Thompson JK. A content analysis of healthy living blogs: evidence of content thematically consistent with dysfunctional eating attitudes and behaviors. Int $\mathrm{J}$ Eat Disord 2014;47(4):362-7.

19. Borzekowski DL, Schenk S, Wilson JL, Peebles R. e-Ana and e-Mia: a content analysis of pro-eating disorder Web sites. Am J Public Health 2010;100(8):1526-34.

20. Custers K, Van den Bulck J. Viewership of pro-anorexia websites in seventh, ninth and eleventh graders. Eur Eat Disord Rev 2009;17(3):214-9.

21. Salomon IP. The selfie generation: examining the relationship between social media use and early adolescent body image [doctoral dissertation]. Lexington (KY): University of Kentucky; 2017.

22. Custers K. The urgent matter of online pro-eating disorder content and children: clinical practice. Eur J Pediatr 2015; 174(4):429-33.

23. Ghaznavi J, Taylor LD. Bones, body parts, and sex appeal: an analysis of \#thinspiration images on popular social media. Body Image 2015;14:54-61.

24. Boepple L, Thompson JK. A content analytic comparison of fitspiration and thinspiration websites. Int J Eat Disord 2016; 49(1):98-101.

25. Tiggemann M, Zaccardo M. "Strong is the new skinny": a content analysis of \#fitspiration images on Instagram. J Health Psychol 2016;1359105316639436.

26. Santarossa S, Coyne P, Lisinski C, Woodruff SJ. \#Fitspo on Instagram: a mixed-methods approach using Netlytic and photo analysis, uncovering the online discussion and author/ image characteristics. J Health Psychol 2016; 1359105316676334.

27. Hester JB, Dougall E. The efficiency of constructed week sampling for content analysis of online news. Journalism Mass Commun Q 2007;84(4):811-24.

28. Harris JK, Mart A, Moreland-Russell S, Caburnay CA. Diabetes topics associated with engagement on Twitter. Prev Chronic Dis 2015;12:E62.

29. Smith M, Milic-Frayling N, Shneiderman B, Mendes Rodrigues E, Leskovec J, Dunne C. NodeXL: a free and open network overview, discovery and exploration add-in for Excel 2007/2010. Redwood City (CA): Social Media Research Foundation; 2010. http://nodexl.codeplex.com/. Accessed November 15, 2017.

\footnotetext{
The opinions expressed by authors contributing to this journal do not necessarily reflect the opinions of the U.S. Department of Health and Human Services, the Public Health Service, the Centers for Disease Control and Prevention, or the authors' affiliated institutions.
} 
30. Harris JK, Moreland-Russell S, Tabak RG, Ruhr LR, Maier RC. Communication about childhood obesity on Twitter. Am J Public Health 2014;104(7):e62-9.

31. Pancer E, Poole M. The popularity and virality of political social media: hashtags, mentions, and links predict likes and retweets of 2016 US presidential nominees' tweets. Soc Influence 2016;11(4):259-70. 


\section{Tables}

Table 1. Characteristics of Relevant \#thinspo and \#fitspo Tweets Collected From Twitter During a Constructed Week ${ }^{\mathrm{a}}$, May 2016

\begin{tabular}{|c|c|c|c|}
\hline \multirow[b]{2}{*}{ Characteristic } & \multicolumn{3}{|c|}{ No. $(\%)^{b}$} \\
\hline & \#thinspo Tweets $(n=458)$ & \#fitspo Tweets $(n=238)$ & All Tweets $(n=696)$ \\
\hline \multicolumn{4}{|l|}{ Engagement with tweet } \\
\hline Retweets, median (IQR) & $20(9-45)$ & $0(0-0)$ & $8(0-31)$ \\
\hline Likes, median (IQR) & $25(9.25-53)$ & $0(0-1.0)$ & $9.5(1-34)$ \\
\hline Replies & $82(17.9)$ & $12(5.0)$ & $94(13.5)$ \\
\hline \multicolumn{4}{|l|}{ Tweet features and topics } \\
\hline Tweet is in English & $397(86.7)$ & $232(97.5)$ & $629(90.4)$ \\
\hline Tweet text is hashtags only & $141(30.8)$ & $21(8.8)$ & $162(23.3)$ \\
\hline Tweet is an advertisement or a fitness/diet tip that appears promotional & $1(0.2)$ & $164(68.9)$ & $165(23.7)$ \\
\hline Tweet includes a link to a website & 0 & $140(58.8)$ & $140(20.1)$ \\
\hline Linked website is about dieting & 0 & $115(48.3)$ & $115(16.5)$ \\
\hline Linked website is about healthy eating & 0 & $3(1.3)$ & $3(0.4)$ \\
\hline Linked website is about physical activity & 0 & $8(3.4)$ & $8(1.1)$ \\
\hline Linked website is about something else & 0 & $18(7.6)$ & $18(2.6)$ \\
\hline Link is broken & 0 & $8(3.4)$ & $8(1.1)$ \\
\hline The tweet includes a mention & $223(48.7)$ & $67(28.2)$ & $290(41.7)$ \\
\hline The tweet includes links to images/videos & $447(97.6)$ & $178(74.8)$ & $625(89.8)$ \\
\hline Images/videos focus on food & 0 & $32(13.4)$ & $32(4.6)$ \\
\hline Images show healthy food & 0 & $19(8.0)$ & $19(2.7)$ \\
\hline Images show unhealthy food & 0 & $2(0.8)$ & $2(0.3)$ \\
\hline Images show a combination of healthy/unhealthy food & 0 & $11(4.6)$ & $11(1.6)$ \\
\hline Images/videos are a body or parts of a body & $435(95.0)$ & $114(47.9)$ & $549(78.9)$ \\
\hline Full body & $243(53.1)$ & $44(18.5)$ & $287(41.2)$ \\
\hline Waist-stomach-hips & $155(33.8)$ & $8(3.4)$ & $163(23.4)$ \\
\hline Torso & $161(35.2)$ & $28(11.8)$ & $189(27.2)$ \\
\hline Neck-shoulders & $71(15.5)$ & $27(11.3)$ & $98(14.1)$ \\
\hline Arms & $36(7.9)$ & $6(2.5)$ & $42(6.0)$ \\
\hline Thighs-legs & $161(35.2)$ & $2(0.8)$ & $163(23.4)$ \\
\hline Butt & $12(2.6)$ & 0 & $12(1.7)$ \\
\hline \multicolumn{4}{|l|}{ Image/video includes one or more person who appears to be ... } \\
\hline Extremely skinny or skeletal & $346(75.5)$ & $8(3.4)$ & $354(50.9)$ \\
\hline Muscular & $3(0.7)$ & 45 (18.9) & $48(6.9)$ \\
\hline
\end{tabular}

Abbreviation: IQR, interquartile range.

${ }^{a}$ Constructed week defined as one Sunday, one Monday, one Tuesday, one Wednesday, one Thursday, one Friday, and one Saturday chosen at random from a specified period of time.

${ }^{\mathrm{b}}$ Unless otherwise indicated.

The opinions expressed by authors contributing to this journal do not necessarily reflect the opinions of the U.S. Department of Health and Human Services, the Public Health Service, the Centers for Disease Control and Prevention, or the authors' affiliated institutions. 
(continued)

Table 1. Characteristics of Relevant \#thinspo and \#fitspo Tweets Collected From Twitter During a Constructed Week ${ }^{\mathrm{a}}$, May 2016

\begin{tabular}{|c|c|c|c|}
\hline \multirow[b]{2}{*}{ Characteristic } & \multicolumn{3}{|c|}{ No. $(\%)^{b}$} \\
\hline & \#thinspo Tweets $(n=458)$ & \#fitspo Tweets $(n=238)$ & All Tweets $(n=696)$ \\
\hline Male only & 0 & $15(6.3)$ & $15(2.2)$ \\
\hline Female only & $435(95.0)$ & $91(38.2)$ & $526(75.6)$ \\
\hline Both & 0 & $7(2.9)$ & $7(1.0)$ \\
\hline Unable to tell & 0 & $1(0.4)$ & $1(0.1)$ \\
\hline \multicolumn{4}{|c|}{ The Twitter user discourages or makes fun of doing something unhealthy or extreme } \\
\hline Yes & $2(0.4)$ & $1(0.4)$ & $3(0.4)$ \\
\hline No & $399(87.1)$ & $230(96.6)$ & $629(90.4)$ \\
\hline Neutral or unable to tell & $57(12.4)$ & $7(2.9)$ & $64(9.2)$ \\
\hline
\end{tabular}

Abbreviation: IQR, interquartile range.

${ }^{a}$ Constructed week defined as one Sunday, one Monday, one Tuesday, one Wednesday, one Thursday, one Friday, and one Saturday chosen at random from a specified period of time.

${ }^{\mathrm{b}}$ Unless otherwise indicated. 
Table 2. Characteristics of 390 Twitter Users Who Tweeted 696 Relevant \#thinspo and \#fitspo Tweets Collected During a Constructed Week ${ }^{\mathrm{a}}$, May 2016

\begin{tabular}{|c|c|c|c|}
\hline Characteristic & \#thinspo Tweets $(n=287$ ) & \#fitspo Tweets $(n=103)$ & All Tweets $(n=390)$ \\
\hline \multicolumn{4}{|l|}{ Category, median (IQR) } \\
\hline Twitter users followed & $212.5(92.25-544.25)$ & $212(77.5-829)$ & $212(88-592)$ \\
\hline Followers of Twitter users & $267(104.25-718.25)$ & $489(132.5-1,299.5)$ & $308(110-870)$ \\
\hline Tweets & $1,591.5(425.25-5,841.75)$ & $3,378(675-17,061.5)$ & $1,794(507-7,105)$ \\
\hline Favorites & $585.5(92.0-2,319.75)$ & $44(3.5-370.5)$ & $363(48-1,745)$ \\
\hline \multicolumn{4}{|l|}{ Type of Twitter user, $n$ (\%) } \\
\hline Individual representing personal views & $286(99.7)$ & $36(35.0)$ & $322(82.6)$ \\
\hline Organization or business & $1(0.3)$ & $56(54.4)$ & $57(14.6)$ \\
\hline Unable to tell & 0 & $11(10.7)$ & $11(2.8)$ \\
\hline
\end{tabular}

Abbreviation: IQR, interquartile range.

${ }^{a}$ Constructed week defined as one Sunday, one Monday, one Tuesday, one Wednesday, one Thursday, one Friday, and one Saturday chosen at random from a specified period of time. 
Table 3. Multilevel Models With the Best Fit for Predicting the Number of Retweets, Likes, and the Probability of a Reply to a Tweet With the \#thinspo or \#fitspo Hashtag on Twitter

\begin{tabular}{|c|c|c|c|c|c|c|}
\hline \multirow[b]{2}{*}{ Characteristic } & \multicolumn{2}{|c|}{ No. of Retweets } & \multicolumn{2}{|l|}{ No. of Likes } & \multicolumn{2}{|c|}{ Probability of Reply } \\
\hline & b (SE) & $P$ Value & b (SE) & $P$ Value & b (SE) & $P$ Value \\
\hline Constant & $-1.22(0.31)$ & $<.001$ & $0.27(0.25)$ & .28 & $-10.80(2.58)$ & $<.001$ \\
\hline \multicolumn{7}{|l|}{ Tweet characteristic } \\
\hline Contains mention & $0.47(0.13)$ & $<.001$ & $0.36(0.13)$ & .004 & $-0.06(0.72)$ & .93 \\
\hline Contains \#thinspo & $3.48(0.31)$ & $<.001$ & $2.38(0.27)$ & $<.001$ & - & - \\
\hline \multicolumn{7}{|l|}{ Sender characteristic } \\
\hline No. of followers, in 100 s & $0.007(0.005)$ & .15 & $0.007(0.005)$ & .16 & $0.004(0.02)$ & .88 \\
\hline No. tweets sent, in 100 s & $-0.0007(0.0002)$ & $<.001$ & $-0.0007(0.0002)$ & $<.001$ & $-0.0004(0.001)$ & .77 \\
\hline \multicolumn{7}{|l|}{ Type of Twitter user } \\
\hline Organization or business & \multicolumn{2}{|c|}{1 [Reference] } & \multicolumn{2}{|c|}{1 [Reference] } & \multicolumn{2}{|c|}{1 [Reference] } \\
\hline Individual representing personal views & $0.85(0.40)$ & .03 & $0.67(0.33)$ & .04 & $1.58(2.51)$ & .53 \\
\hline Unable to tell & $0.52(0.48)$ & .27 & $0.03(0.40)$ & .94 & $-0.33(4.37)$ & .94 \\
\hline
\end{tabular}

Abbreviation: SE, standard error.

${ }^{a}$ Models are based on 574 tweets and 335 tweet senders. 